\section{Christoph Bachmann}

\section{Die Cochrane Collaboration}

Die CC ist eine internationale gemeinnützige Organisation, die sich an den Grundsätzen der evidenzbasierten Medizin orientiert und zur Verbesserung der wissenschaftlichen Grundlagen von Behandlungen beitragen möchte (Abb. 1). Dazu gibt die CC regelmässig systematische Übersichtsarbeiten (Metaanalysen) auf der Basis von kontrollierten klinischen Studien in Auftrag. Die CC wurde 1993 gegründet und ist nach dem britischen Epidemiologen Sir Archibald Leman Cochrane benannt, dessen Wirken die evidenzbasierte Medizin beeinflusst hat. Zur Erstellung eines Reviews werden externe Fachleute beauftragt.

\section{Der Review über Pelargonium}

Im Jahre 2013 erschien ein Review der CC mit dem Titel «Pelargonium sidoides extract for treating acute respiratory tract infections» [1]. Darin wurde also die Wirksamkeit von Präparaten aus P. sidoides zur Behandlung von akuten Atemwegsinfekten untersucht. Diese Metaanalyse wurde von einem Autorenkollektiv unter der Leitung von Antje Timmer durchgeführt.

\section{Design}

In die Studie aufgenommen wurden alle identifizierten doppelblinden, randomisierten und kontrollierten

\title{
Extrakt aus Pelargonium sidoides zur Behandlung akuter Infektionen der Atemwege: Ein Cochrane-Review bestätigt (und relativiert) die Wirksamkeit
}

Wenn immer wieder klinische Studien über die Wirksamkeit eines Medikamentes oder einer Behandlungsmethode bei bestimmten Erkrankungen publiziert werden, dann gibt bald einmal die Cochrane Collaboration (CC) einem Autorenteam den Auftrag, einen sogenannten Cochrane-Review zu publizieren, in dem die Wirksamkeit dieser Therapiemethode untersucht wird. Dies ist natürlich auch bei pflanzlichen Arzneimitteln der Fall. In der Folge wird ein Cochrane-Review vorgestellt, welcher die Wirksamkeit von Extrakten aus der südafrikanischen Pflanze Pelargonium sidoides untersucht.

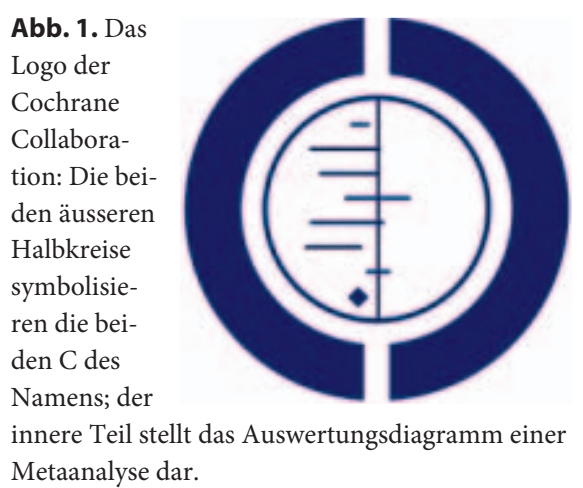

Abb. 1. Das Logo der Cochrane Collaboration: Die beiden äusseren symbolisieren die beiNamens; de Metaanalyse dar.

Studien, welche die Wirksamkeit von P.-sidoides-Präparaten bei akuten Atemwegsinfekten gegenüber Placebo oder einer anderen Behandlungsmethode untersuchten.

Als primärer Endpunkt wurde das vollständige Verschwinden der Symptome definiert. Ausserdem wurde das Verschwinden folgender SchlüsselSymptome untersucht:

- Bronchitis: Husten, produktiver Husten, Sputum-Bildung;

- Tonsillitis oder Pharyngitis: Schluckbeschwerden, Halsschmerzen;

- Laryngitis: Heiserkeit;

- Rhinitis und Sinusitis: Nasensekretion, Kopfschmerzen;
- Erkältung: Halsschmerzen, verstopfte Nase;

- Varia: Fieber, Unwohlsein.

\section{Auswahl und Beurteilung der Studien}

Folgende Studien nahmen die Cochrane-Autoren in den Review auf und beurteilten diese:

Bachert et al., 2009 [2]

- Design: RCT (randomisierte kontrollierte Studie), doppelblind, Multizenter.

- Probanden: 103; Diagnose: akute bakterielle Sinusitis.

- Behandlung: Alkoholischer Wurzelextrakt von $P$. sidoides, $3 \times 60$ Tropfen, versus Placebo während 60 Tagen.

- Endpunkte primär: Veränderung im Symptomscore nach 7 Tagen.

- Endpunkte sekundär: Veränderung des Schweregrades individueller Symptome am Tag 7 und 21, Lebensqualität, Beurteilung durch Prüfärzte, unerwünschte Ereignisse.

\section{KARGER}

Fax +497614520714
๑ 2017 S. Karger GmbH, Freiburg 
- Resultat: Pelargonium (EPs 7630) besser als Placebo, gute Verträglichkeit.

Chuchalin et al., 2005 [3]

- Design: RCT, doppelblind, Multizenter.

- Probanden: 124; Diagnose: akute Bronchitis.

- Behandlung: Alkoholischer Wurzelextrakt von $P$. sidoides, $3 \times 30$ Tropfen versus Placebo während 7 Tagen.

- Endpunkte primär: Veränderung im Symptomscore nach 7 Tagen.

- Endpunkte sekundär: Veränderung des Schweregrades individueller Symptome, Beurteilung durch Patienten und Prüfärzte, unerwünschte Ereignisse.

- Resultat: Pelargonium (EPs 7630) besser als Placebo.

\section{Kamin et al., 2010 [4]}

- Design: RCT, doppelblind, Multizenter.

- Probanden: 200 (Kinder); Diagnose: akute Bronchitis.

- Behandlung: Alkoholischer Wurzelextrakt von $P$. sidoides, $3 \times 30$ Tropfen (bzw. altersabhängig) versus Placebo während 7 Tagen.

- Endpunkte primär: Veränderung im Symptomscore nach 7 Tagen.

- Endpunkte sekundär: Veränderung des Schweregrades individueller Symptome, Beurteilung durch Patienten und Prüfärzte, unerwünschte Ereignisse.

- Resultat: EPs 7630 ist Placebo gegenüber überlegen und gut verträglich.

\section{Kamin et al., 2010 [5]}

- Design: RCT, Dosis-Bestimmung, dreiarmig;

- Probanden: 405 Kinder; Diagnose: akute Bronchitis;

- Behandlung: P.-sidoides-Tabletten 10/20/30 mg versus Placebo während 7 Tagen.

- Endpunkte primär: Veränderung im Symptomscore nach 7 Tagen.

- Endpunkte sekundär: Veränderung des Schweregrades individueller
Symptome, Beurteilung durch Patienten und Prüfärzte, unerwünschte Ereignisse.

- Resultat: 60 oder 90 mg Pelargonium (EPs 7630) verbessern die Symptome signifikant besser als Placebo, gute Verträglichkeit.

\section{Kamin et al., 2012 [6]}

- Design: RCT, doppelblind, Multizenter.

- Probanden: 220 Kinder; Diagnose: akute Bronchitis.

- Behandlung: P.-sidoides-Tropfen $3 \times$ 10-30 Tropfen (altersabhängig) versus Placebo während 7 Tagen.

- Endpunkte primär: Veränderung im Symptomscore nach 7 Tagen.

- Endpunkte sekundär: Veränderung des Schweregrades individueller Symptome, Beurteilung durch Patienten und Prüfärzte, unerwünschte Ereignisse.

- Resultat: Pelargonium (EPs 7630) wirksam und gut verträglich.

Lizogub et al., 2007 [7]

- Design: Doppelblind, Multizenter.

- Probanden: 103; Diagnose: Erkältung.

- Behandlung: Alkoholische P.-sidoidesTropfen, $3 \times 30$ Tropfen versus Placebo, während 7 Tagen.

- Endpunkte primär: Unterschied auf dem ErkältungssymptomeScore an den Tagen 1 und 5.

- Endpunkte sekundär: Totales Verschwinden der Symptome, Veränderung des Schweregrades individueller Symptome, Beurteilung durch Patienten und Prüfärzte, unerwünschte Ereignisse.

- Resultat: Pelargonium (EPs 7630) verbessert die Symptome signifikant mehr als Placebo, gute Verträglichkeit.

\section{Matthys et al., 2007 [8]}

- Design: RCT, doppelblind, Multizenter.

- Probanden: 217; Diagnose: akute Bronchitis.

- Behandlung: Alkoholische P.-sidoides-Tropfen, $3 \times 30$ Tropfen versus Placebo während 7 Tagen.
- Endpunkte primär: Veränderung im Symptomscore nach 7 Tagen.

- Endpunkte sekundär: Veränderung des Schweregrades individueller Symptome, Beurteilung durch Patienten und Prüfärzte, unerwünschte Ereignisse.

- Resultat: Pelargonium (EPs 7630) ist verglichen mit Placebo sehr wirksam und gut verträglich.

Matthys und Heger, 2010 [9]

- Design: RCT, Dosis-Bestimmung, dreiarmig.

- Probanden: 399; Diagnose: akute Bronchitis.

- Behandlung: P.-sidoides-Tabletten 10/20/30 mg versus Placebo während 7 Tagen.

- Endpunkte primär: Veränderung im Symptomscore nach 7 Tagen.

- Endpunkte sekundär: Veränderung des Schweregrades individueller Symptome, Beurteilung durch Patienten und Prüfärzte, unerwünschte Ereignisse.

- Resultat: Alle 3 Dosen sind Placebo gegenüber signifikant überlegen, gute Verträglichkeit.

\section{Beurteilung der Cochrane- Autoren}

Es ist eine Tatsache, dass Reviews der CC, welche die Wirksamkeit von pflanzlichen Arzneimitteln untersuchen, die untersuchten Studien äusserst streng beurteilen und sehr häufig die methodische Qualität der Publikationen in Zweifel ziehen. Dies ist auch bei dem vorliegenden Review der Fall. So schreiben die Autoren bei der Beurteilung der Resultate:

«Auf der Basis der zur Verfügung stehenden Studiendaten kann der Extrakt aus Pelargonium sidoides zum Beheben der Symptome einer akuten Bronchitis bei Erwachsenen und Kindern als wirksam bezeichnet werden. Die statistische Heterogenität wurde teilweise durch die Verwendung verschiedener Präparate erklärt. In Bezug auf den Heilungsgrad nach 7 Tagen erwiesen sich nur die Tropfen als wirksam. Die Tabletten erwiesen sich nicht als wirksam. Bei den alkoholischen Extrakten bestand eine Übereinstimmung bei den Resultaten 
in Bezug auf alle Symptome und die Schlüsselsymptome. Aber beim alkoholischen Extrakt gab es pro Altersgruppe nur je zwei Studien und bei den Tabletten nur je eine mehrarmige Dosisfindungsstudie.

Gesamthaft betrachtet ist die Qualität der Evidenz für diese Resultate gering.»

Diese letzte Aussage begründen die Autoren unter anderem mit der teilweise mangelhaften Verblindung und Randomisierung, den verwendeten Skalen der Symptome sowie der Interpretation der Resultate und auch mit der geringen Anzahl von Probanden, mit der einige der Studien durchgeführt wurden.

\section{Replik}

Gleichermassen ist bekannt, dass Autoren von Cochrane-Reviews sehr häufig keine wirklichen Phytotherapie-Fachleute sind. So haben die Autoren des vorliegenden Reviews allenfalls nicht realisiert, dass auch in den Studien, in denen Tabletten mit $P$. sidoides eingesetzt wurden, diese Tabletten als Wirkstoff einen (abgedampften) alkoholischen Extrakt aus P. sidoides enthielten.

\section{Literatur}

1 Timmer A, Günther J, Motschall E, Rücker G, Antes G, Kern WV: Pelargonium sidoides extract for treating acute respiratory tract infections. Cochrane Database Syst Rev 2013;(10): CD006323.

2 Bachert C, Schapowal A, Funk P, Kieser M: Treatment of acute rhinosinusitis with the preparation from Pelargonium sidoides EPs 7630: a randomized, double-blind, placebocontrolled trial. Rhinology 2009;47:51-58.

3 Chuchalin AG, Berman B, Lehmacher W: Treatment of acute bronchitis in adults with a Pelargonium sidoides preparation (EPs 7630): a randomized, double blind, placebo-controlled trial. Explore 2005;1:437-445.
Gemäss den Autoren des Reviews erwiesen sich die Tabletten als nicht wirksam. Wenn man aber die Resultate der entsprechenden Studie betrachtet, dann erhält man ein ganz anderes Bild: Dort wird für jede Dosis (30, 60 bzw. $90 \mathrm{mg}$ ) zwischen Baseline und dem Tag 7 gegenüber Placebo eine signifikante Verminderung des «Bronchitis Symptom Score» ermittelt ( $\mathrm{p}<0,0001$ für jede Dosierung). Auch die sekundären Endpunkte zeigten ähnliche Resultate. Der Leiter dieser Studie, Prof. Heinrich Matthys, ist ein ausgewiesener Fachmann auf dem Gebiet der Phytotherapie, der sich jahrelang mit pflanzlichen Anwendungen in der Pneumologie beschäftigt hat.

\section{Fazit}

Der vorliegende Review der CC untersucht die Wirksamkeit von P.-sidoides-Extrakten zur Behandlung von akuten Infekten der Atemwege. Dafür wurden 8 Studien ausgewertet, von denen 2 Studien mehrarmig waren und je 3 verschiedene Dosierungen untersuchten. Alle Studien wurden mit dem standardisierten P.-sidoidesExtrakt EPs 7630 durchgeführt. Diese wichtige Tatsache erwähnen die Autoren in ihrer Metaanalyse nicht.

In einem ersten Schritt beurteilen die Autoren die Wirksamkeit als positiv, relativieren dann aber diese Aussagen mit vielen Kritikpunkten an der Methodik der Studien. Die Studie von Matthys et al. [8] wird als unwirksam bezeichnet, obwohl diese für alle 3 untersuchten Dosierungen gegenüber Placebo eine signifikante Wirksamkeit aufzeigt.

Wahrscheinlich könnte bei jeder Studie eine methodische Schwäche gefunden werden. Deshalb gilt hier, was schon am Anfang des Kapitels «Beurteilung der Cochrane-Autoren» gesagt wurde.

Zusammenfassend kann gesagt werden, dass der P.-sidoides-Extrakt EPs 7630 zur Behandlung einer akuten Bronchitis und der anderen in den 8 ausgewerteten Studien überprüften Indikationen wirksam und sicher ist. Damit bietet sich das Präparat in Form von Tropfen oder Tabletten als Behandlungsoption bei entsprechenden Erkrankungen an.
4 Kamin W, Maydannik V, Malek FA, Kieser M: Efficacy and tolerability of EPs 7630 in children and adolescents with acute bronchitis - a randomized, double-blind, placebo-controlled multicenter trial with a herbal drug preparation from Pelargonium sidoides roots. Int J Clin Pharmacol Ther 2010;48:184-191.

5 Kamin W, Maydannik VG, Malek FA, Kieser M: Efficacy and tolerability of EPs 7630 in patients (aged 6-18 years old) with acute bronchitis. Acta Paediatr 2010;99:537-543.

6 Kamin W, Ilyenko LI, Malek FA, Kieser M: Treatment of acute bronchitis with EPs 7630: randomized, controlled trial in children and adolescents. Pediatr Int 2012;54:219-226.

7 Lizogub VG, Riley DS, Heger M: Efficacy of Pelargonium sidoides preparation in patients with the common cold: a randomized, double blind, placebo-controlled clinical trial. Explore 2007;3:573-584.
8 Matthys H, Heger M: Treatment of acute bronchitis with a liquid herbal drug preparation from Pelargonium sidoides (EPs 7630): a randomised, multicentre, double-blind, placebo-controlled study. Curr Med Res Opin 2007;23:323-331.

9 Matthys H, Lizogub VG, Malek FA, Kieser M: Efficacy and tolerability of EPs 7630 tablets in patients with acute bronchitis: a randomised, double-blind, placebo-controlled dose-finding study with a herbal drug preparation from $\mathrm{Pe}$ largonium sidoides. Curr Med Res Opin 2010; 26:1413-1422. 